\title{
Erratas y correcciones manuscritas de autor en la traducción latina de la Política de Aristóteles de Juan Ginés de Sepúlveda editada por Michel de Vascosan (París, 1548)
}

\author{
Julián Solana Pujalte \\ Universidad de Córdoba \\ ca1sopuj@uco.es \\ Ignacio J. GARCíA PINILLA \\ Universidad de Castilla-La Mancha \\ Ignacio.GPinilla@uclm.es
}

Recibido: 25 de octubre de 2011

Aceptado: 15 de diciembre de 2011

\section{RESUMEN}

La edición de Michel de Vascosan de la traducción latina de la Política de Aristóteles de Juan Ginés de Sepúlveda (París, 1548) apareció con un gran número de erratas. Esta circunstancia molestó profundamente a Sepúlveda que tomó nota de todas ellas y de sus correspondientes correcciones en dos ejemplares diferentes, destinados a servir de base a una nueva edición que nunca llegó a ver la luz y que son el objeto del presente trabajo.

Palabras clave: Juan Ginés de Sepúlveda. La Política de Aristóteles. Aristoteles latinus. Michel de Vascosan. Crítica textual.

Solana Pujalte, J. - García Pinilla, I.J., «Erratas y correcciones manuscritas de autor en la traducción latina de la Política de Aristóteles de Juan Ginés de Sepúlveda editada por Michel de Vascosan (París, 1548)», Cuad. Fil. Clás. Estud. Lat. 31.2 (2011) 293-309.

\section{Errata and authorial handwritten corrections in Michel de Vascosan's edition of the Latin translation of Aristotle's Politics by Juan Ginés de Sepúlveda (Paris, 1548)}

\begin{abstract}
This paper studies the large number of misprints in Michel de Vascosan's edition of the Latin translation, by Juan Ginés de Sepúlveda, of Aristotle's Politics (Paris, 1548). The author, deeply disappointed, marked them in two different copies. They were planned to be used in the preparation of a new edition of the work, never printed.
\end{abstract}

Keywords: Juan Ginés de Sepúlveda. Aristotle’s Politics. Aristoteles latinus. Michel de Vascosan. Textual criticism.

Solana Pujalte, J. - García Pinilla, I.J., «Errata and authorial handwritten corrections in Michel de Vascosan's edition of the Latin translation of Aristotle's Politics by Juan Ginés de Sepúlveda (Paris, 1548)», Cuad. Fil. Clás. Estud. Lat. 31.2 (2011) 293-309.

Sumario 1. La génesis de la edición. 2. Las correcciones de las erratas. 3. Edición de las correcciones. 4. Referencias bibliográficas. 


\section{LA GÉNESIS DE LA EDICIÓN}

El año 1548 veía la luz en las prensas parisinas de Michel de Vascosan la traducción latina acompañada de escolios de la Política de Aristóteles de Juan Ginés de Sepúlveda. Era el fruto maduro de muchos años de trabajo del Sepúlveda helenista, que contaba con una nutrida relación de traducciones de obras aristotélicas y de sus comentaristas publicadas en tierras italianas y francesas bastantes años antes, entre 1522 y 1532 :

1. Parvi naturales. Bononiae, per Hieronymum de Benedictis, 1522, 15 de mayo.

2. De generatione et interitu. Bononiae per Hieronymum de Benedictis, 1523, 15 de mayo.

3. De mundo. Bononiae per Hieronymum de Benedictis, 1523, 20 de agosto.

4. Comentarios de Alejandro de Afrodisias a la Metafísica de Aristóteles. Romae, in aedibus Marcelli Silber, alias Franch, 1527, mense februario.

5 Meteorum libri. Lutetiae, Ioannes Parvus, 15321.

Una obra tan compleja como esa era el fruto de un largo proceso de estudio y elaboración, que se remontaba casi quince años atrás. Por una carta de 13 de febrero de 1534 enviada a Gian Matteo Giberti ${ }^{2}$ sabemos que Sepúlveda había empezado la traducción latina de la Política, pero que la postergó a la paraphrasis Ethicorum que le había sugerido Giberti por iniciativa de Clemente VII, cuyos dos primeros libros tenía concluidos un año más tarde ${ }^{3}$. El periodo de elaboración de la traducción y los comentarios llega hasta 1546, es decir dura unos doce años, pues conocemos por una carta suya de 1 de septiembre de ese mismo año, que había escrito poco tiempo atrás a Simon de Colines para intentar llegar a un acuerdo con el afamado impresor parisino de cara a la edición de su traducción de la Política ${ }^{4}$. Sepúlveda había otorgado poderes a un amigo, Diego de Carvajal, para ejecutar el posible acuerdo, pero el fallecimiento de Colines ese mismo año desbarató los planes. A pesar de ello, Carvajal debió de gestionar con celeridad el encargo editorial de Sepúlveda, pues muy poco tiempo después (17.XI.1546) Sepúlveda comunicaba por carta a Jerónimo Zurita que Carvajal había llegado a un acuerdo con «otro que había habido las letras mesmas del muerto y tenía noticia de mí y había ofrecido de imprimir la Política y scholia a su costa y aun darme a mí trescientos volúmines, con pagarle yo solamente el papel d'ellos» ${ }^{5}$. Finalmente sería Michel de Vascosan el encargado de llevar a término la empresa en 1548. El resultado fue lamentable: una edición hermosa por sus caracteres, pero plagada de erratas. Si eso en cualquier libro es una tragedia, en una traducción latina del griego, y de una obra como la Política aristotélica, en la que la precisión es fundamental y de la

\footnotetext{
${ }^{1}$ Sobre Sepúlveda helenista, vid. Green (1940); Losada (1948); Rodríguez Peregrina (1984); Coroleu (1993), (1994a), (1994b), (1995), (1996a), (1996b), (1997), (2009); Solana Pujalte (1998), (2000); Valverde Abril (2006a), (2006b); Vollet (2002).

${ }^{2}$ La 26 de nuestra edición del Epistolario sepulvediano, por la que citamos: García Pinilla - Solana Pujalte (2007, IX, pp. 72-73).

${ }^{3}$ Carta 31 de 20.II.1535, ibid., p.85.

${ }^{4}$ Carta 63 de 1.IX.1546, ibid., p. 159

${ }^{5}$ Carta 64 de 17.XI.1546, ibid., pp. 160-161. El impresor aludido es Regnault de Chaudière. Se conserva el contrato firmado entre este impresor y Carvajal, que no llegó a ejecutarse. Vid. A. Parent (1974, pp.101-102).
} 
que para colmo acababan de editarse dos nuevas traducciones ${ }^{6}$, la reiteración de erratas de todo tipo, muchas enormemente burdas, suponía un golpe de muerte. Sepúlveda lo sabía perfectamente. Por eso el año siguiente a la publicación dirigía a Vascosan una carta seca y exenta de toda cortesía en la que se mostraba muy molesto con la negligencia del impresor (nec tuam vigilantiam magnopere desiderarem nisi inter initia nonnihil dormitasses) y con el gran número de erratas (menda non pauca) de la edición y le comunicaba que si se decidía a imprimir de nuevo el libro, dada la buena acogida que estaba teniendo, podría enviarle una relación de todas las erratas que había detectado ${ }^{7}$. Sabemos que Vascosan no volvió a editar la obra, pero también que Sepúlveda no echó el asunto en el olvido, pues en febrero de 1554 enviaba a Francisco de Argote otra copia de sus propias correcciones. Ignoramos el motivo de tal envío, pero la referencia de pasada a las erratas deja a las claras su malestar por lo sucedido ${ }^{8}$.

Sepúlveda procuró por todos los medios que la edición parisina de 1548 no fuera la definitiva de la traducción que tantos desvelos le había costado, el opus maius de su labor como helenista, e intentó que Paolo Manuzio la imprimiera de nuevo en Venecia. Parece ser que Manuzio se había ofrecido y Sepúlveda le envió un ejemplar del libro corregido, pero no sabemos por qué no llegó a editarse ${ }^{9}$.

Según Juan Gil, Sepúlveda era un perfeccionista y un maniático de las correcciones ${ }^{10}$. Aparte de la reelaboración constante a que sabemos que sometía a sus obras ${ }^{11}$, procuraba subsanar las erratas de las ya publicadas. Recordemos que se puso en contacto con el mismo Vascosan en 1550 para proponerle imprimir una fe de erratas de sus Opera que había impreso en 1541, nueve años antes, Simon de Colines, sin ocul-

\footnotetext{
${ }^{6}$ La de Jacques-Louis d'Estrebay (Strebaeus), profesor del colegio de Sainte-Barbe de París, en la imprenta de Vascosan; y la de Joachim Périon (Perionius), benedictino profesor de Teología en París, en la imprenta de Tiletano. Sobre ellos, vid. Ch. H. Lohr (1988, pp.438 y 322-324), respectivamente; Grente - Pauphilet - Pichard (eds.) (1951, pp. 316 y 564) y Maillard - Kecskeméti - Magnen - M. Portalier (1999, pp.121-162 y $349-479)$.

${ }^{7}$ Carta 83 de 1.VIII.1549, ibid., p.248.

${ }^{8}$ Carta 112 de 9.II.1554. Errata quibis librarii opus Aristotelis De republica a me conversum et enarratum multis in locis depravarunt et emendationes cum his ad te litteris mitto (ibid., p.323).

${ }^{9}$ Carta 130 de 1.XI.1561. Entresacamos algunos párrafos: Libros Aristotelis De republica in latinum sermonem per me e Graeco in Latinum sermonem conversos et scholiis enarratos, Lutetiae Vascosanus bonis quidem characteribus... sed satis negligenter excusit. Hos ego nuper nonnihil otii nactus recognovi et queadam loca in melius mutavi... Scripserat ad me Sebastianus Leo noster audivisse ex te, cum idem opus affirmares libenter te Aldinis tuis, id est optimis typis, diligenter mandaturum, si opus recognitum et emendatum nactus esses: qua de re cum ipse multos ante menses ad te scripserim litterasque in fasciculo Veneti legati perferendas dederim, librumque correctum detulerim, nihildum tamen a te litterarum accepi. Excudendi opus in Gallia occasionem confido equidem non defuturam, mihi tamen gratissimum esset, scripta mea tui nominis tuorumque typorum auctoritate tuaque diligentia exornari, modo id tibi nec molestum sit nec inutile, ut simul vetus nostra necessitudo novis officiis renovetur. Tuum igitur erit, ut me de tua voluntate quam primum certiorem facias ut, si hoc tibi placere cognovero, librum ad te correctum et cum qua dixi coronide primo quoque tempore per Venetum tabellarium mittendum curem... (ibid., p.384). J. Gil apunta a que probablemente no interesara a Manuzio editar un libro que hacía años que circulaba ya por Europa (ibid., I,p.XCI).

${ }^{10} \mathrm{Ibid}$., I,pp.LXXXVIII-XCI.

${ }^{11}$ Vid. Solís de los Santos (2010, cap. IV: «La transmisión del texto: realidad y conjetura", especialmente «La incansable revisión de los apógrafos”, pp.CLV-CLVI); Bellido Díaz (2008, pp.195-248); Solana Pujalte - García Pinilla, (en prensa).
} 
tarle que estaba en tratos simultáneamente con Gaspar Trechsel para que emprendiera la misma tarea ${ }^{12}$.

Es imaginable, pues, el interés puesto por Sepúlveda en la revisión y corrección de las erratas de la edición parisina y en su ulterior publicación, lo que lamentablemente no llegó a ver concluido. Los desvelos correctores de Sepúlveda, plasmados en las erratas anotadas sobre el texto de la edición de 1548, quedaron en el olvido hasta que los académicos de la Real de la Historia, con motivo de la edición en 1780 de los Opera cum edita tum inedita de Sepúlveda, encontraron un ejemplar de la edición de Vascosan, con las correcciones de las erratas a las que hace referencia Sepúlveda en su correspondencia ${ }^{13}$, y las editaron en el vol. ${ }^{14}$. Recientemente hemos localizado otro ejemplar que también contiene las correcciones manuscritas de Sepúlveda ${ }^{15}$.

\section{LAS CORRECCIONES DE LAS ERRATAS}

En este trabajo hemos decidido colacionar las correcciones de ambos ejemplares, poniéndolas en relación con la edición de las mismas que realizaron los académicos a partir del ejemplar de la Real Academia de la Historia y con la edición parisina de Vascosan de 1548. Facilitará esta colación -al menos eso pretendemos- la tarea de edición de la traducción sepulvediana de la Política, pues aporta la que es sin duda la última revisión de la obra por parte de su autor ${ }^{16}$.

Las erratas que se deslizaron en el texto fueron muy numerosas (más de 630 son las correcciones según nuestros datos), por lo que no pueden tener cabida todas ellas en este trabajo. Nos contentamos con incluir únicamente las correcciones al texto de la traducción, dejando para otra ocasión las que afectan a los escolios.

Hemos apreciado dos manos diferentes en las correcciones de ambos impresos. Una primera, que llamaremos «mano 1», la más frecuente en ambos, presenta una escritura de pequeño tamaño y de un trazo regular, probablemente corresponde a un amanuense. La escritura de la «mano 2» es de mayor tamaño y de trazo algo más irregular y vacilante; usa tinta más oscura y ferruginosa; corresponde, a nuestro entender, a la letra del propio Sepúlveda que encontramos de vez en cuando en algunos de sus manuscritos ${ }^{17}$. De esta mano son todos los añadidos marginales que identifican algunas de las fuentes del texto, p.e. los que aparecen en los ff. $97,113 \mathrm{v}, 152 \mathrm{v}, 153 \ldots$ estos añadidos solo aparecen en el ejemplar de la catedral de Córdoba y corresponden casi siempre a los escolios.

\footnotetext{
${ }^{12}$ Carta 90 de 7.VII.1550 (ibid., IX.2 p.258).

${ }^{13}$ Biblioteca de la Real Academia de la Historia, 2/8292.

${ }^{14}$ La edición de las erratas en Sepúlveda, Juan Ginés de (1780, pp.CXIII-CXLIII). Sobre la edición, vid. Gil (1975, pp.93-129), reeditado en Id. (1984, pp.127-162).

${ }^{15}$ Biblioteca de la Catedral de Córdoba, 832, antigua signatura est. 6 caj. $3 \mathrm{n}^{\circ}$ 8. Carta 83 (ibid., p.248, nota 427).

${ }^{16}$ Está muy avanzada la edición crítica de esta obra que preparan F. Arenas-Dolz y M. Herrero de Jaúregui como volumen XVI de la edición pozoalbense de las Obras completas de Juan Ginés de Sepúlveda. Agradecemos a los editores que nos hayan proporcionado una primera redacción de su trabajo.

${ }^{17}$ Puede verse reproducida en Losada (1984, lámina VI, margen superior derecho) y en Bellido Díaz (2008, pp.200-206), donde se reproduce la letra de varias correcciones interlineales de Sepúlveda en los manuscritos de la obra refererida.
} 
Cuando se trata de pequeñas correcciones de una o varias letras o textos muy pequeños resulta más difícil distinguir las manos, por lo que solo las indicamos en los textos de una cierta extensión, suficientes, en cualquier caso, para su clara identificación.

\begin{tabular}{|c|c|c|}
\hline Folio & $\mathbf{C}^{18} \quad(\mathrm{BCC} 832)$ & M $\quad$ (BRAH 2/8292) \\
\hline $14 \mathrm{v}$ & Mano 1 & Mano 1 \\
\hline $15 \mathrm{v}$ & Mano 1 & Mano 1 \\
\hline $21 \mathrm{v}$ & Mano 1 & Mano 1 \\
\hline 24 & Mano 1 & Mano 2 \\
\hline 34 & Mano 2 & Mano 1 \\
\hline 43 & Mano 2 tachada por mano 1 & Mano 2 \\
\hline 52 & Mano 1 & Mano 1 \\
\hline $58 \mathrm{v}$ & Mano $1 \mathrm{mg}$. Mano 2 tachada inter lineas & Mano 1 \\
\hline 59 & Mano 1 & Mano 1 \\
\hline $67 \mathrm{v}$ & Mano 2 & Mano 2 \\
\hline 68 & Mano 2 & $\begin{array}{l}\text { Mano 2: el texto griego de la primera } \\
\text { nota y las dos notas siguientes. } \\
\text { Mano 1: el texto latino de la } \\
\text { primera nota }\end{array}$ \\
\hline 71 & Mano 1 & Mano 1 \\
\hline 77 & Mano 1 & $\begin{array}{l}\text { Mano 1. Mano 2: solo "quendam" } \\
\text { inter lineas 10-11 }\end{array}$ \\
\hline 79 & Mano 1 & Mano 1 \\
\hline $80 \mathrm{v}$ & Mano 1 & Mano 1 \\
\hline 83 & Mano 1 & Mano 1 \\
\hline 99 & $\begin{array}{l}\text { Mano 2: Corrección interlineal y } 5 \text { primeras } \\
\text { líneas de la nota marginal. } \\
\text { Mano 1: Desde la línea } 6 \text { al final de la nota } \\
\text { marginal. (Vid. lámina 1) }\end{array}$ & Mano 1 \\
\hline 107 & $\begin{array}{l}\text { Mano 1: primera nota marginal. } \\
\text { Mano 2: segunda nota. }\end{array}$ & Falta \\
\hline $107 \mathrm{v}$ & Mano 1 & Mano 1 \\
\hline $111 \mathrm{v}$ & Mano 2 & Mano 1 \\
\hline 117 & Mano 1 & Mano 1 \\
\hline $126 \mathrm{v}$ & Mano 1 & Mano 1 \\
\hline $127 \mathrm{v}$ & Mano 2 & Mano 1 \\
\hline 130 & Mano 1. Mano 2: añadido final del escolio. & Mano 1 \\
\hline 141 & $\begin{array}{l}\text { Mano 1: al margen. Mano 2: al final del } \\
\text { escolio inter lineas }\end{array}$ & Mano 1 \\
\hline $176 \mathrm{v}$ & Mano 1 & Mano 1 \\
\hline 205 & Mano 2 & Mano 1 \\
\hline 240 & Mano 1 & Mano 1 \\
\hline
\end{tabular}

${ }^{18}$ C: Biblioteca de la Catedral de Córdoba. M: Biblioteca de la Real Academia de la Historia. Vid. infra. 
¿Son anteriores las correcciones del ejemplar cordobés o las del matritense? ¿Se puede determinar si unas son copia de las otras? Una de las diferencias más notables entre $\mathrm{C}$ y $\mathrm{M}$ son los añadidos que sirven para precisar mejor las referencias a autores y obras que aparecen en el texto, casi siempre de los escolios, y que solo presenta C. Son añadidos, no correcciones de erratas, que introduce Sepúlveda para enriquecer el texto e identificar con precisión las referencias. P.e. f. Aiijv, 18 De finibus lib. 3 $m g$. add. C; 56v,21 in Ages. $m g$. add. C; 58v,24 Plut. in Lyc. $m g$. add. C; 67v,15 in Sol. et Marc. Cat. $m g$. add. C; 68,15 Plut. In Sol. mg. add. C etc. Se podría pensar que al ser solo $\mathrm{C}$ quien tiene estos añadidos sería posterior a $\mathrm{M}$, y el proceso de copia habría sido $\mathrm{M}=>\mathrm{C}$, pero también estos paratextos podrían haberse añadido a $\mathrm{C}$ después del proceso inverso $\mathrm{C}=>\mathrm{M}$.

Esporádicamente hay alguna corrección que presenta $\mathrm{C}$ o $\mathrm{M}$ y no el otro impreso, pero son aisladas, no obedecen a un patrón determinado, y quizá solo se deban a descuidos de la copia, por lo que tampoco puede sacarse de ellas ninguna conclusión.

El único indicio que, en nuestra opinión, permitiría defender la anterioridad de $\mathrm{C}$ sobre $\mathrm{M}$ y la posible dependencia de este último del primero, estriba en una serie de correcciones realizadas en $\mathrm{C}$, que por ser erróneas o inexactas no prosperaron y fueron sustituidas después por otras diferentes in situ en C. En M no aparecen nunca las primitivas correcciones de $\mathrm{C}$, por lo que parece que $\mathrm{M}$ tomó directamente las formas definitivas de C. El que solo C presente esas primeras correcciones fallidas, además siempre de la mano de Sepúlveda, parece indicar que las correcciones de C son anteriores a las de $\mathrm{M}$ y que por tanto directa o indirectamente las segundas han sido tomadas de las primeras. No existe el mismo fenómeno en M. Veamos esos casos ${ }^{19}$ :

59, 30 Camicum em. CMa : Camerinum mg. eras. C : Caminum p

$79 \mathrm{v}, 11$ recte vivendi partem nactus est $\mathbf{C a}$ : recti vivendi partem natus est $\mathbf{M}$ : quatenus cuique partem adiicit recte vivendi. ut. quatenus cuique auget recte vivendi facultatem $m g$. eras. $\mathbf{C}$ : potest recte vivendi particeps esse $\mathbf{p}$

$111 \mathrm{v}, 27$ equorum quos alunt $\mathbf{C M}$ : ex equis quos alunt $i$. l. eras. $\mathbf{C}:$ aequorum alendorum $\mathbf{p}$

172,8 Artabanes CM : Artabanus mg. eras. C : Artapanes p

190v,30 potentias em. CMa : potentiam i.l. eras. C : potentiae $\mathbf{p}$

225,4-5 obtineat em. CMa : obtineant mg. eras. $\mathbf{C}$ : obtinent $\mathbf{p}$

Como es natural, las intervenciones más numerosas realizadas por Sepúlveda sobre el texto tienen por objeto restaurar el original subsanando las erratas que se habían deslizado en el impreso. Sepúlveda, no obstante, aprovecha la ocasión, como hemos visto ya en otras obras (Solana Pujalte-García Pinilla: en prensa, nota 11), para introducir ocasionalmente mejoras gramaticales, léxicas y estilísticas e identificar con mayor precisión algunas de las fuentes mencionadas. Los casos que hemos identificado son estos:

\footnotetext{
${ }^{19}$ Para las siglas, vid. infra.
} 


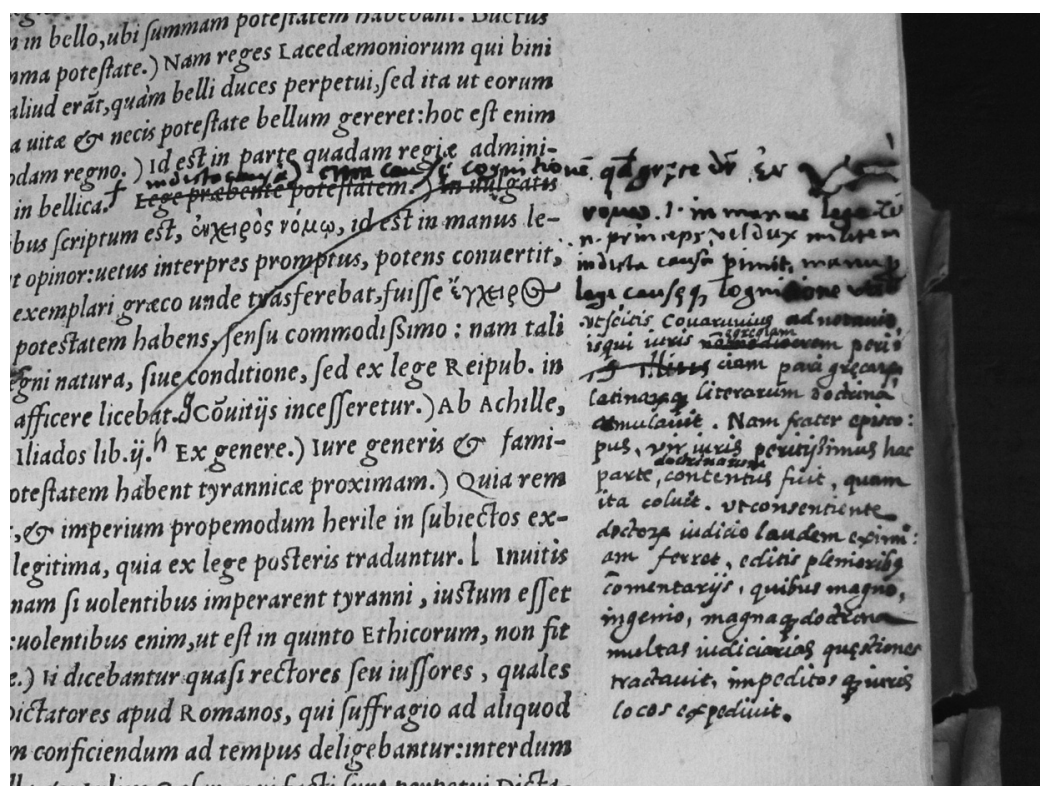

Lámina I. Biblioteca de la Catedral de Córdoba, 832, f.99.

41,17 interire em. CMa : mittere p; 45v,17 Pars manet ignauum par fortiter arma cienti $\mathrm{em}$. CMa : Improbus atque probus pariter donantur honore p; 66,28-29 adoptione $\mathrm{em}$. CMa : procreatione p; 97v,16 indicta causa $\mathrm{em}$. CMa : lege prebente potestatem $\mathbf{p}$; 123v,2324 Quoniam igitur em. CMa : Est igitur causa, quoniam p; 123v,26-27 constare dicunt em. CMa : videtur constare p; 128,10 elementis em. CMa : principiis p; 144,6 iniquum em. CMa : inaequale p; 148,21 simul habitantibus em. CMa : inquilinis p; 241v,24 pueris em. CMa : adolescentibus p; 243v,10 accommodanda em. CMa : dirigenda p y 127v,31-128,5, de mayor extensión, a cuyo texto remitimos infra ${ }^{20}$.

Se debieron de hacer varias copias de estas correcciones. Tenemos noticias de la prometida a Vascosan si se decidía a editar una nueva y de las enviadas a Francisco de Argote y a Paolo Manuzio ${ }^{21}$. Naturalmente Sepúlveda tendría alguna más en su poder. Es lógico pensar que las dos copias que nos han llegado, dado que se encuentran en bibliotecas a las que fueron a parar libros que pertenecieron a Sepúlveda, estuvieran destinadas a su propio uso por el autor ${ }^{22}$.

\footnotetext{
${ }^{20}$ La identificación de las fuentes solo se produce en los escolios introducidos por Sepúlveda para glosar la obra, por lo que se analizarán en un trabajo aparte dedicado específicamente a ellos.

${ }^{21} \mathrm{Vid}$. notas 6,7 y 8.

${ }^{22}$ Sepúlveda donó a la catedral de Córdoba sus libros griegos, vid. Losada (1973 [1949], p.518), donde se trascribe su testamento; Nieto Cumplido (1979, pp.745-750), donde se relacionan los libros actualmente conservados. A la Biblioteca de la Real Academia de la Historia fue a parar material muy diverso a raíz de las pesquisas de los académicos para la edición de sus Obras Completas, en especial los códices de las Crónicas de Carlos V y Felipe II, vid. Gil $(1975,1984)$.
} 
Queremos dejar constancia, por último, de que hemos encontrado dos estados diferentes en la edición parisina de Vascosan de 1548:

1. En el f.255v., línea 26 estaba impreso al inicio de la línea: «ertamina pertinent». Percatados del error en la imprenta de Vascosan, se corrigió en «certamina pertinēt», manteniéndose el resto de la línea y de la página igual. Presentan el estado p1 (con la errata original) el ejemplar de la Biblioteca de la Real Academia de la Historia (2/8292) y el de la Universidad de Barcelona (07 CM-3634), y el p2 (con la errata corregida) los ejemplares de la Biblioteca de la Catedral de Córdoba (832), la Biblioteca Nacional de España (R/25106[1]), Biblioteca de la Universidad de Granada (BHR/A-005260), la Biblioteca Pública de Córdoba (24/120), Bibliothèque Nationale de France (NUMM-54644) y Bayerische Staatsbibliothek (4.A.gr.b.449).

2. Otro caso similar es el del f. 98, línea 30: el primer texto salido de las prensas es «geroicorum». La errata es detectada y se corrige por «heroicorum». Presentan el estado p1 los ejemplares de la Biblioteca de la Real Academia de la Historia, la Biblioteca de la Universidad de Barcelona, la Biblioteca Pública de Córdoba y la Bibliothèque Nationale de France y el p2 (corregido ya en imprenta) los ejemplares de la Biblioteca de la Catedral de Córdoba, Biblioteca de la Universidad de Granada, Biblioteca Nacional de España y Bayerische Staatsbibliothek.

No deja de ser paradójico que estas insignificantes erratas de impresión, sin repercusión alguna sobre el contenido, fueran detectadas y enmendadas por los revisores y no los centenares que señalaremos más abajo, muchas de las cuales afectan al sentido del texto, que escaparon inexplicablemente a la revisión.

\section{EDICIÓN DE LAS CORRECCIONES}

Las siglas usadas son: ta, 1780 .

a : Editio Academica emendationum. Matriti, ex Typographia Regia De la Gaze-

p : Editio Parisiensis, apud Vascosanum, 1548.

p1 Errata typographica aliquibus in exemplaribus contenta nec emendata (vide paulum supra)

p2 Emendationes typographicae eorundem erratorum aliis in exemplaribus (vide paulum supra)

M : Emendationes manu scriptae in exemplari Bibliothecae Regiae Historicae Academiae Matritensis (2/8292).

C : Emendationes manu scriptae in exemplari Bibliothecae Capitularis Cordubensis (832, olim est. 6 caj. 3 n 8 ).

La primera cifra de las referencias da cuenta del folio y la segunda de la línea en los que se encuentran las correcciones ${ }^{23}$.

\footnotetext{
${ }^{23}$ Usamos em. (emendavit/emendaverunt) para referirnos a las correcciones manuscritas $\mathbf{C}$ y $\mathbf{M}$ у om. (omisit) para referirnos a los casos en que la editio academica (a) omite las correcciones introducidas por C o M.
} 
in titulo Parisiis Apud Vascosanum...annos $\mathbf{p}$ : eras. $\mathbf{M} \|$ paratum ab ipso auctore ad aliam editionem uidetur add. M || in verso tituli Privilegii sententia...ex consilio Guiot eras. M

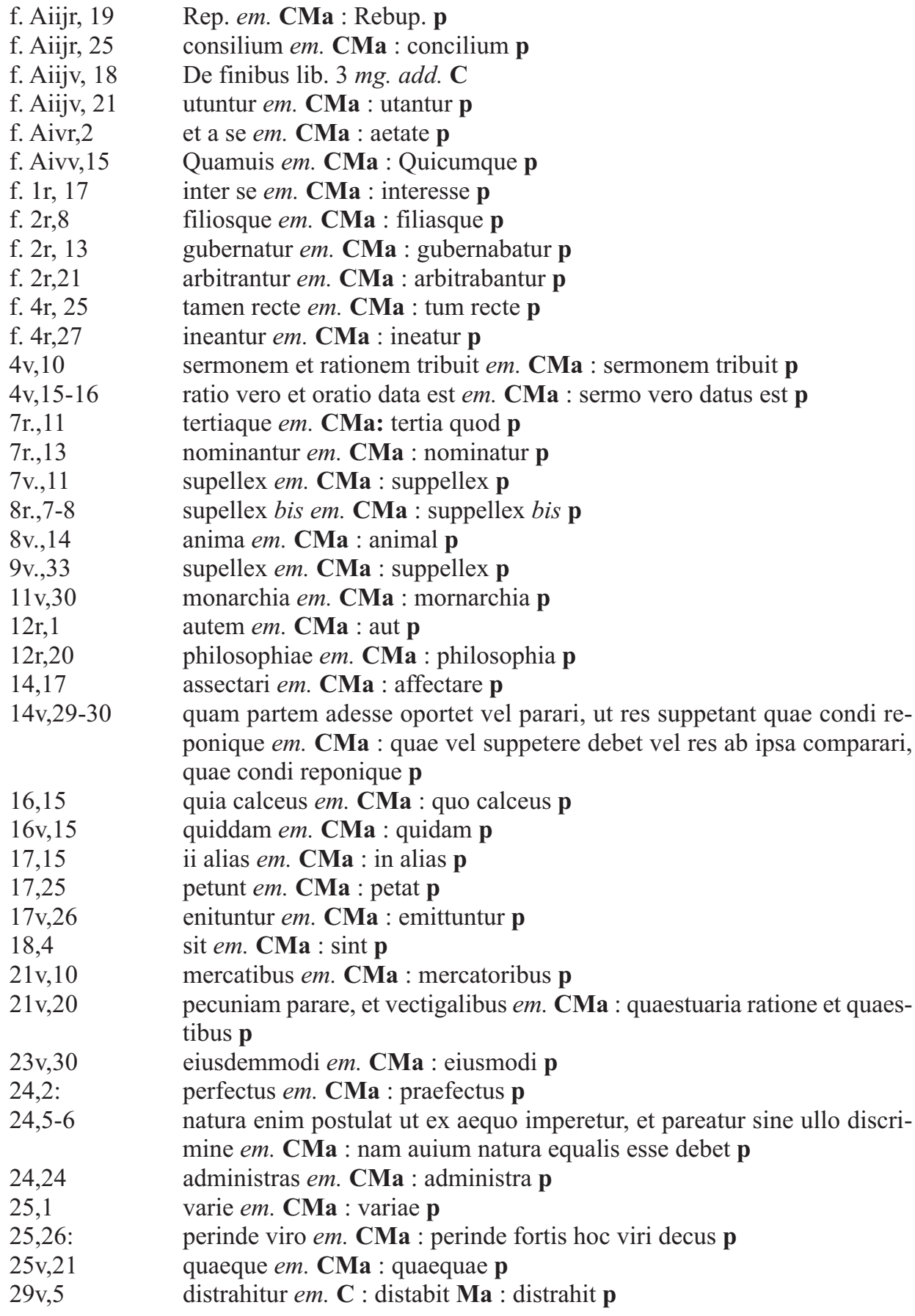




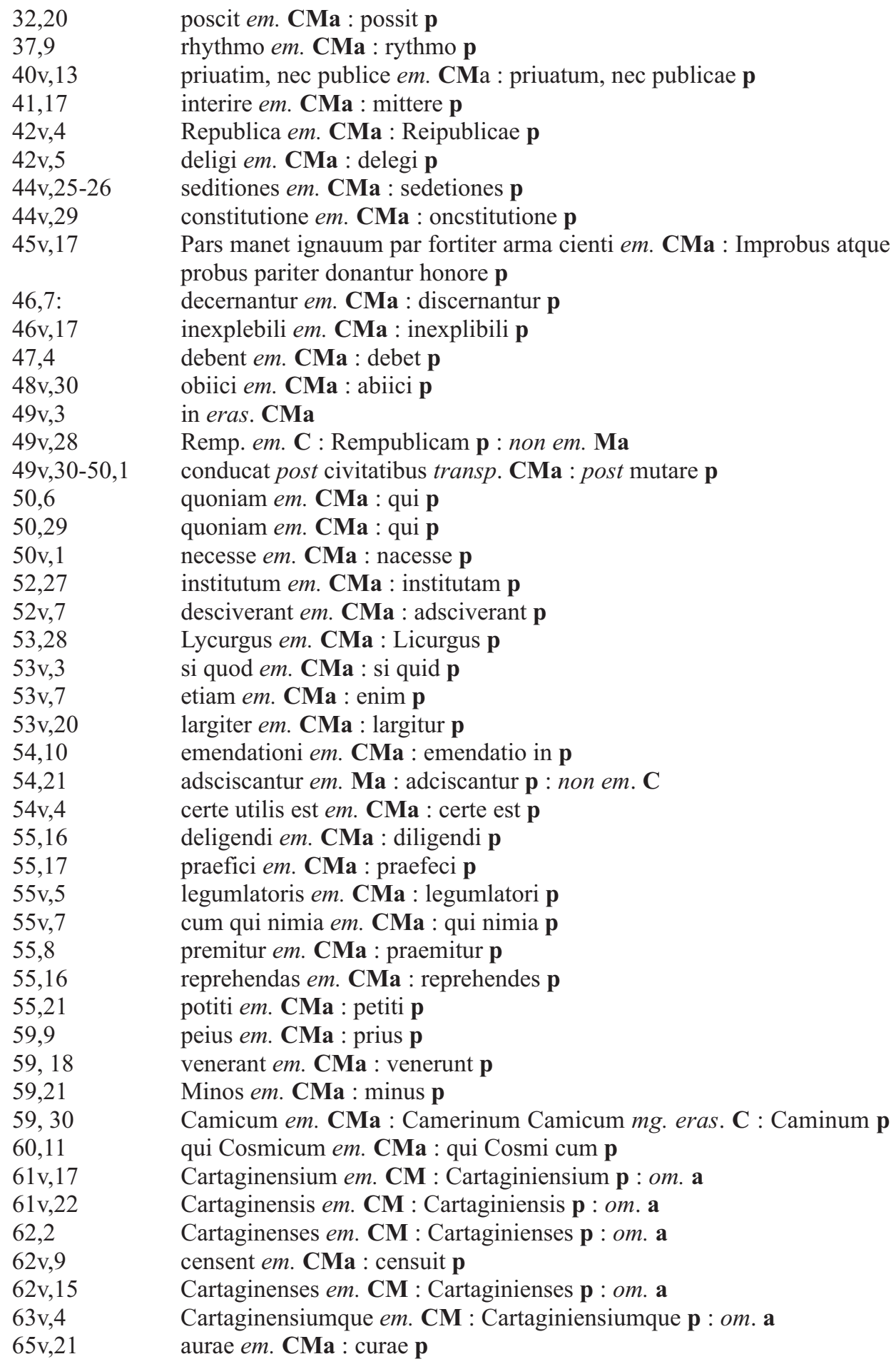




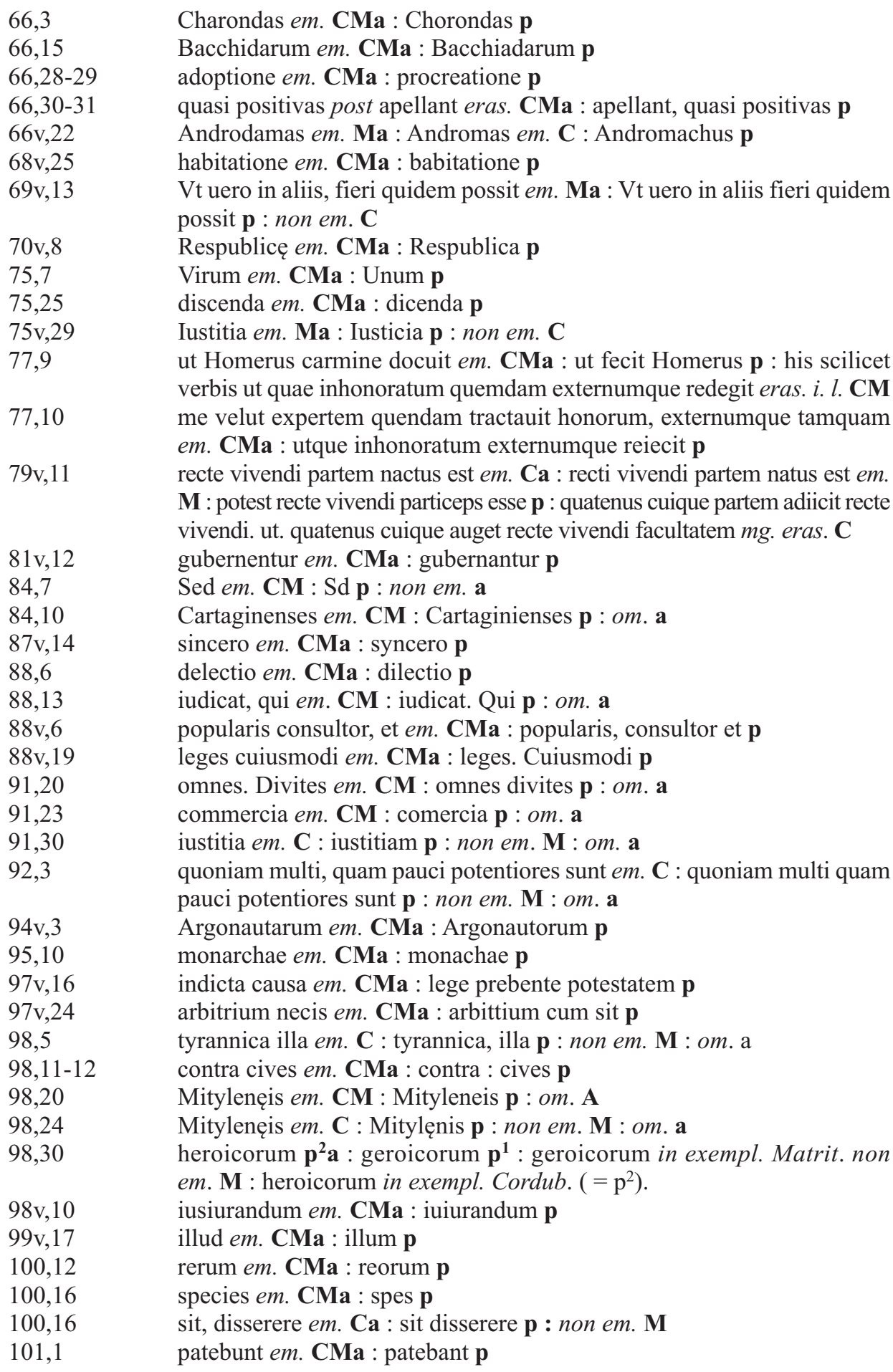


101,17

101,18

103,26

$103,26-27$

$103,27-28$

103,33

104,14

104,23

105,14

105,16

$105 \mathrm{v}, 5$

$105 \mathrm{v}, 21$

108,19

108,26

110,28

110,31

$110 v, 18$

111,3

111,4

111,9

$111 \mathrm{v}, 27$

$113 \mathrm{v}, 9$

$113 \mathrm{v}, 24$

$113 \mathrm{v}, 27$

$113 \mathrm{v}, 29$

114,2

115,3

116,8

116,26

119,32

120,29

$120 \mathrm{v}, 16$

121,4

121,7

$122 \mathrm{v}, 2$

$122 \mathrm{v}, 23$

coepere em. a : cepere $\mathbf{C M}$ : caepere $\mathbf{p}$

hinc, cum em. $\mathbf{C a}$ : hinc cum $\mathbf{M}$ : huic cum $\mathbf{p}$

parte. Sed. em. CMa : parte, sed $\mathbf{p}$

dicitur (hoc em. CMa : dicitur. Hoc p

moderatur), quibusdam em. CMa : moderatur, quibusdam $\mathbf{p}$

perniciosum em. CMa : pernitiosum $\mathbf{p}$

cum em. $\mathbf{C M}$ : eum $\mathbf{p}:$ om. $\mathbf{a}$

si vadant simul em. CMa : congressi p

ut ferat multitudinem et ingeniorum imperia ab his qui $\mathrm{em}$. $\mathbf{C}$ : ut ferat imperantem multitudinem, possitque ingeniorum imperio ab his qui $\mathbf{p}$ : non em. $\mathbf{M}:$ om. a

gubernari. post civile transp. CMa : post excellunt $\mathbf{p}$

praestare $\mathrm{em}$. CMa : praeferri pcm

administratione em. CMa : administrationem $\mathbf{p}$

diutissime em. CMa : diuitissime $\mathbf{p}$

plerique em. CMa : plerisque $\mathbf{p}$

aliud, quam em. $\mathbf{C M}$ : aliud quam $\mathbf{p}: \mathrm{om}$. a

quid, inter $\mathrm{em}$. $\mathbf{C M}$ : quid inter $\mathbf{p}: \mathrm{om}$. $\mathbf{a}$

optima paucorum potentia $\mathrm{em}$. $\mathbf{C M}$ : optimam paucorum potentiam $\mathbf{p}$ : om. a

velit, has em. Ma : velit has $\mathbf{p}:$ non $\mathrm{em}$. C

Rursusque paucorum potentias postremo, cum breuiter de his omnibus, quatenus licebit, memorauerimus $\mathrm{em}$. M : Rursusque paucorum potentias, postremo, cum breuiter de his omnibus, quatenus licebit, memorauerimus em. C : Rursusque paucorum potentias. Postremo, cum breviter de his omnibus, quatenus licebit, memoraverimus $\mathrm{em}$. a : Rususque paucorum potentias, postremo cum breuiter de his omnibus, quatenus licebit, breui memorauerimus $\mathbf{p}$

potissimae $\mathrm{em}$. $\mathbf{C}$ : potissime $\mathbf{p}$ : non em. $\mathbf{M}$ : om. a

equorum quos alunt CMa : ex equis quos alunt $i$. l. eras. $\mathrm{C}:$ aequorum alendorum $\mathbf{p}$

Nam eo quidem em. CMa : Eoquidem $\mathbf{p}$

paucorum potentia $m g$. add. $\mathbf{C M}$

Lydiis em. CM : Lydis $\mathbf{p}$ : om. a

sint em. CMa : sunt $\mathbf{p}$

sint em. CMa : sunt $\mathbf{p}$

ut iidem em. CMa : ut idem $\mathbf{p}$

ut cuivis em. CMa : ut cuius $\mathbf{p}$

precio em. $\mathbf{C M}$ : pretio a : praecio $\mathbf{p}$

census em. CMa : sensus p

consultandum necesariis $\mathrm{em}$. $\mathbf{C M}$ : consultandum, necesariis $\mathbf{p}: \mathrm{om}$. a vectigalibus $\mathrm{em}$. CMa : vestigalibus $\mathbf{p}$

vivant em. CMa : vinant $\mathbf{p}$

pauciores, qui em. $\mathbf{C}$ : pauciores qui $\mathbf{p}:$ non em. $\mathbf{M}: \mathrm{om}$. a

oligarchiam em. CMa : obligarchiam $\mathbf{p}$

In his tamen sunt quidam, quos fama commendat em. M : In his tamen sunt quidam quos fama commendat $\mathrm{em}$. $\mathbf{C}$ : Sunt tamen quidam quos fama commendat $\mathbf{p}: \mathrm{om}$. $\mathbf{a}$ 


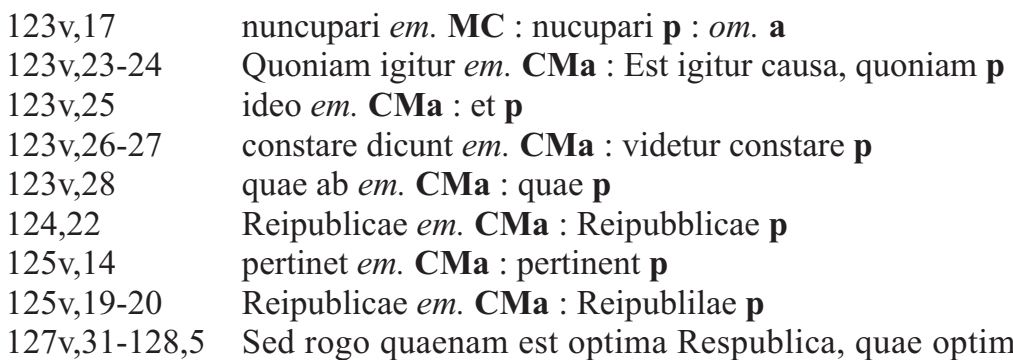
civitatibus, et plerisque hominibus, non ad egregiam virtutem, nec ad institutionem, quae res naturam adiumentaque fortunae desiderant, nec ad Rempublicam, quae optari posset, spectantibus, sed ad vitam, quae plerisque communis esse valeat, et ad Rempublicam, qua uti queant pleraeque (plereque M) civitates em. CMa : Quae vero sit optima Respublica, quae optima vita plerisque civitatibus, et plerisque hominibus qui non aegregia virtute, nec doctrina, quae res naturam adiumentaque fortunae desiderant, Reipublicae vivendique rationem metiuntur, nec dirigunt ad normam eius quae optari posset Reipublicae, sed vitam quaerunt quae hominum maiori parti possit esse communis, et Rempublicam qua civitatum magna pars uti posset $\mathbf{p}$

128,8 utraque em. CMa : utrisque p

128,10 elementis em. CMa : principiis p

$129 \mathrm{v}, 8$

131,28

Respublicae em. CM : Respublica p : om. a

$131 \mathrm{v}, 26$

superentur em. CMa : superantur p

$132 \mathrm{v}, 19$

Rempublicam em. CMa : Respublica p

133,11 iudicia obierint em. CMa : indicia obierint p

133,15

134,30

$134 \mathrm{v}, 22$

$143 v, 29$

144,6

147,26

$147 \mathrm{v}, 3$

$147 \mathrm{v}, 6$

$147 \mathrm{v}, 13$

$147 \mathrm{v}, 17$

$147 \mathrm{v}, 22$

148,21

$150 \mathrm{v}, 28$

Respublica em. CMa : Reipublicae p

simpliciter em. CMa : simplibiter $\mathbf{p}$

Respublicae em. CMa : Respulica p

Republica, vel ut em. Ca : Republica vel ut em. M : Republica velut p

Heliaeam em. CMa : Helisam p

iniquum em. CMa : inaequale $\mathbf{p}$

administraretur em. CMa : administretur $\mathbf{p}$

mutationes, quoniam em. CM : mutationes. Quoniam $\mathbf{p}$ : om. a

duobus em. CMa : duabus p

Iapygibus em. a : Iapigibus $\mathbf{p}:$ non em. $\mathbf{C M}$

concisi em. CMa : conscisi $\mathbf{p}$

quidem, accidit tamen em. CMa : quidem accidit, tamen $\mathbf{p}$

simul habitantibus em. CMa : inquilinis p

puellam locupletem orbam em. Ma : puellam locupletem $\mathbf{C}$ : puellam etiam orbam $\mathbf{p}$

151v,11 in omni em. CMa : nisi p

155v,12-13 obiirent em. $\mathbf{C}$ : obirent $\mathbf{p}:$ non em. a

$155 \mathrm{v}, 24$

suffragio em. CMa : saffragio $\mathbf{p}$

$159 \mathrm{v}, 11$

fortitudine em. CMa : a fortitudine $\mathbf{p}$

$163,4-5$

instituta $\mathrm{em}$. $\mathbf{C M}$ : institua $\mathbf{p}: \mathrm{om}$. a

164,12

opulentis em. CMa : oppulentis p

$164 \mathrm{v}, 21$

sanciendum em. $\mathbf{a}$ : santiendum $\mathbf{p}$ : non em. $\mathbf{C M}$ 


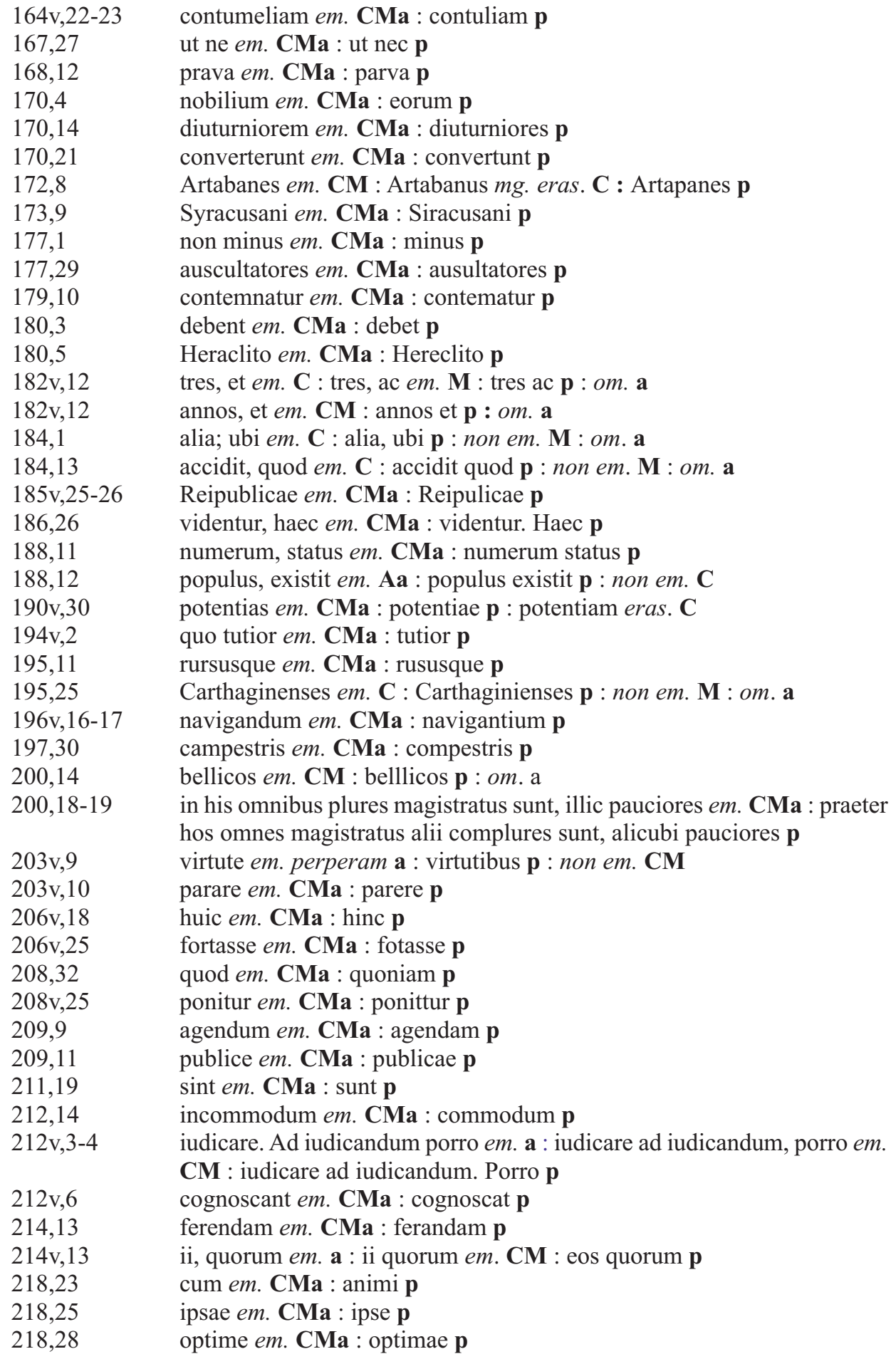




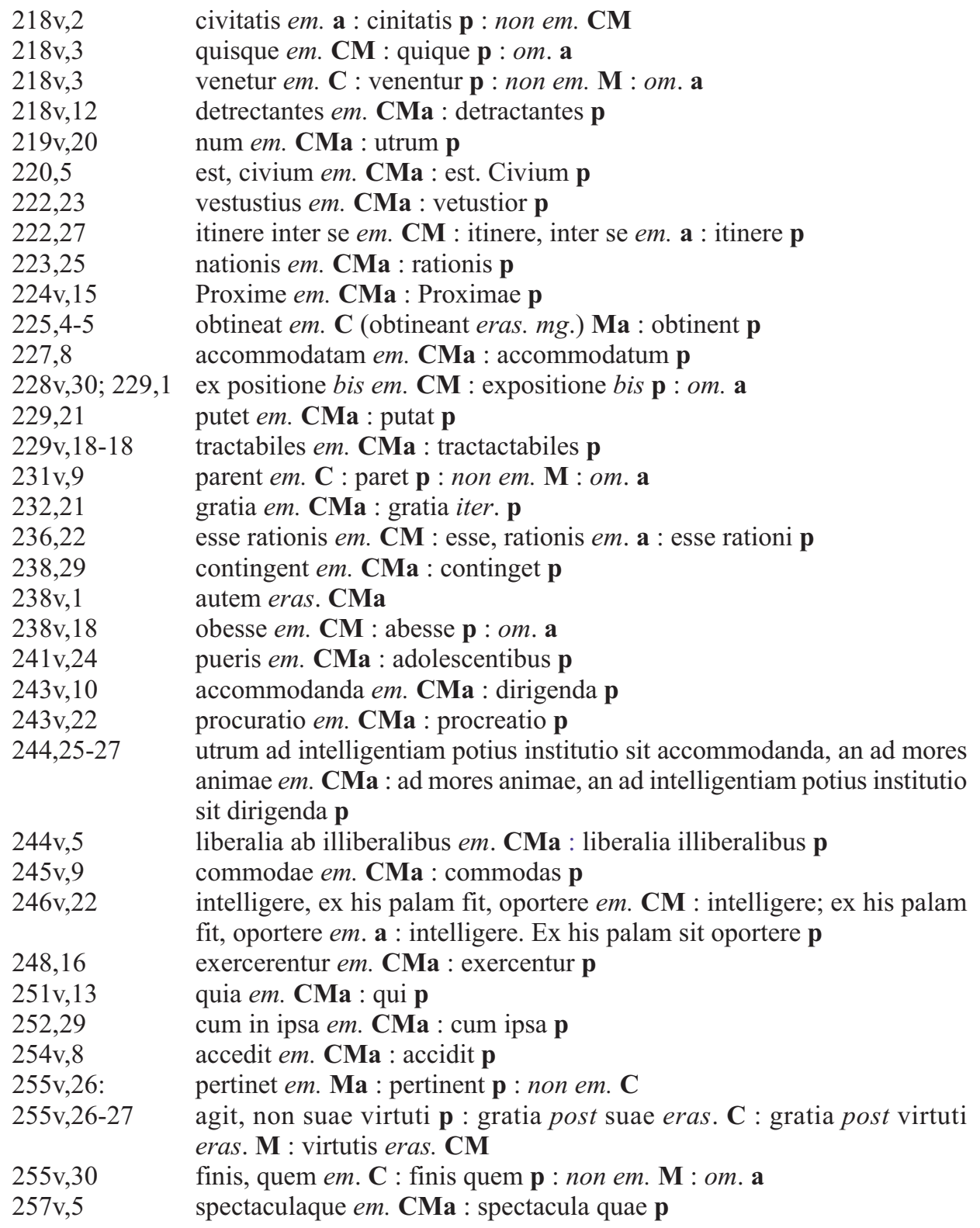

\section{REFERENCIAS BIBLIOGRÁFICAS}

Bellido DíAz, J. A. (2008), «Indefessae labor limae Sepuluedanae en los libros XIX y XX de la De rebus gestis Caroli Quinti Historia», Exemplaria Classica 12, 195-248.

Coroleu, A. (1993), Estudios sobre Juan Ginés de Sepúlveda: sus traducciones latinas de Aristóteles, Tesis doctoral en microfichas, Universidad de Barcelona, 1993. 
Coroleu, A. (1994a), «La contribución de Juan Ginés de Sepúlveda a la edición de los textos de Aristóteles y de Alejandro de Afrodisias», HL 43, 231-245.

Coroleu, A. (1994b), «Le glosse di Juan Ginés de Sepúlveda alle traduzioni latine di Aristotele», Giornale Critico della Filosofia Italiana 75,1, 16-32.

Coroleu, A. (1995), «A philological Analysis of Juan Ginés de Sepúlveda’s Latin Translations of Aristotle and Alexander of Aphrodisias», Euphrosyne n.s. 23, 175-195.

Coroleu, A. (1996a), «Ioannes Genesius Sepulueda uersus Franciscus Vatablus. A propósito de la fortuna de las traducciones latinas de Juan Ginés de Sepúlveda», Habis 27, 277-281.

Coroleu, A. (1996b), «The Fortuna of Juan Ginés de Sepúlveda’s Translations of Aristotle and of Alexander of Aphrodisias», JWCI 59, 325-332.

Coroleu, A. (1997), «De la didáctica al diálogo: cita y uso de Aristóteles en el Democrates Secundus de Juan Ginés de Sepúlveda», en J.Ma Maestre Maestre., J. PAscual BareA, L. Charlo Brea (coords.), Humanismo y pervivencia del mundo clásico. Homenaje al profesor Luis Gil (Alcañiz, del 2 al 6 de mayo de 1995), Alcañiz - Cádiz, I, pp.129-136.

Coroleu, A. (2009), «Notas críticas (y casi contemporáneas) a la traducción de Juan Ginés de Sepúlveda de la Política aristotélica», Euphrosyne n.s. 37, 375-379.

García Pinilla, I. J. - Solana Pujalte, J. (2007), Juan Ginés de Sepúlveda, Obras Completas, VIII, IX.1, IX.2, Epistolario. Edición crítica, traducción e introducción filológica de I. J. GARCía Pinilla, J. Solana Pujalte. Introducción histórica de J. Gil, Pozoblanco, 2007.

GiL, L. (1975), «Una labor de equipo: la Editio Matritensis de Juan Ginés de Sepúlveda», CFC 8, 93-129.

GIL, L. (1984), Estudios de Humanismo y tradición clásica, Madrid, 1984

Green, O.H. (1940), «A note on Spanish Humanism. Sepúlveda and his Traslation of Aristotle's Politics», Hispanic Review 8, 339-342.

Grente, G. - Pauphilet, A. - Pichard L. (eds.) (1951), Dictionnaire des Lettres françaises. Le seizième siècle, Paris, 1951.

Lohr, Ch. H. (1988), Aristotle Commentaries, 2. Renaissance Authors, Firenze.

LosADA, A. (1948), «Juan Ginés de Sepúlveda, traductor y comentarista de Aristóteles», Revista de Filosofía 7, 499-536.

LosadA, A. (1973 [1949]) ,Juan Ginés de Sepúlveda a través de su «Epistolario» y nuevos documentos, Madrid.

LosadA, A. (1984 [1951]), Juan Ginés de Sepúlveda, Demócrates Segundo o de las justas causas de la guerra contra los indios. Edición y traducción de A. Losada, Madrid.

Maillard, J.-F. - Kecskeméti, J. - Magnen, C. - Portalier, M., (1999), La France des Humanistes. Hellenistes I, Turnhout.

Nieto Cumplido, M. (1979), «Fondos librarios de Juan Ginés de Sepúlveda en la Biblioteca de la Catedral de Córdoba», Studia Albornotiana 37, 745-750.

Parent, A., (1974), Les métiers du livre à Paris au XVI siècle, Genève.

Rodríguez PeregrinA, E. (1984), «Juan Ginés de Sepúlveda y sus traducciones comentadas de los filósofos griegos», Estudios de Filología Latina 4, 235-246.

SEPÚlvedA, JuAn GINÉS DE (1780), Joannis Genesiii Sepuluedae Cordubensis Opera, cum edita, tum inedita, accurante Regia Historiae Academia uolumen primum. Matriti, ex Typographia Regia de la Gazeta, anno M.DCC.LXXX.

Solana Pujalte, J. (1998), «Una colección única de impresos de Juan Ginés de Sepúlveda en la Biblioteca de la Universidad Pontificia de Comillas», en Homenaje a Esperanza Albarrán Gómez, Sevilla, pp.183-191. 
Solana Pujalte, J. (2000), «Los Errata Petri Alcyonii in interpretatione libri Aristotelis de incessu animalium de Juan Ginés de Sepúlveda: ¿obra quemada, no impresa o no publicada?» en Acta Conuentus Neo-Latini Abulensis. Proceedings of the tenth International Congress of Neo-Latin Studies (Ávila, 4-9 August 1997), Tempe, 597-602.

Solana Pujalte, J. - García Pinilla, I. J., (en prensa), «Postrema lima: anotaciones manuscritas inéditas de Sebastián de León, secretario de Juan Ginés de Sepúlveda, al diálogo Democrates siue de honestate disciplinae militaris (Roma, 1535)», Neulateinisches Jahrbuch.

Solís De los Santos, J. (2010), Juan Ginés de Sepúlveda, Obras Completas XIV. Historia de Carlos V. Libros XXVI-XXX. Ed. crítica, traducción, introducción filológica, notas e índice de J. Solís de los Santos. Estudio histórico de B. Cuart Moner, Pozoblanco.

VAlVerde Abril, J.J. (2006a), «Una notable página en la historia de la filología: los Aristotelis de republica libri VIII de Juan Ginés de Sepulveda», en XII International Congress for Neo-latin Studies. Latin as the International Language of Scholarship from the Renaissance to the Present (Bonn, 3-9 August 2003), Tempe, pp.847-860.

VAlverde Abril, J.J. (2006b) «Las traducciones latinas de la Politica de Aristóteles: Panorama general», Calamus Renascens 7, 197-215.

Vollet, M. (2002), «Sepúlveda: traductor y comentarista de Aristóteles, Política I», Ideas y valores $119,137-143$. 